\title{
Los estudios doctorales en Europa y su armonización ¿realidad o utopía?
}

Doctoral Studies in Europe and their Harmonization. Reality or Utopia?

\section{Gustavo Eduardo Toledo Lara*}

(iD) https://orcid.org/0000-0002-5104-9555

Tipo de Artículo: Informes de Investigación y ensayos inéditos

Doi: 10.17533/udea.unipluri.20.2.014

Toledo Lara, G. (2020). Los estudios doctorales en Europa y su armonización ¿realidad o utopía? UniPluriversidad, 20(2), e20202014. doi: 10.17533/udea.unipluri.20.2.014

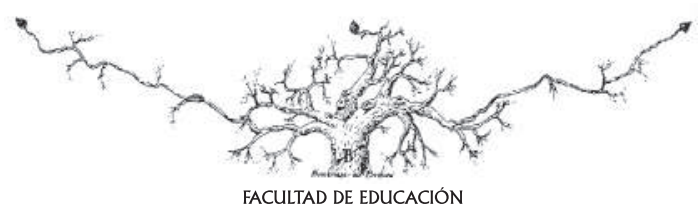

Recibido: 2020-04-22 • Aprobado: 2020-12-22

* Universidad Camilo José Cela. España.

Email: gustavotoledolara@gmail.com 


\title{
Resumen
}

En este artículo se presenta una investigación sobre el doctorado en Europa con el objeto de analizar su armonización según los criterios orientadores impulsados desde el Espacio Europeo de Educación Superior (EEES). La armonización se expresa en el reconocimiento de los estudios universitarios a partir de orientaciones procedimentales para la administración curricular asumidos por todos los Estados signatarios. Sin embargo, se observan algunas diferencias: forma de considerar a los estudiantes, duración de estudios, o evaluación. Este estudio se realiza desde una perspectiva cualitativa para inferir, analizar e interpretar el sentido de los documentos. El estatus de los matriculados en el doctorado y la evaluación de estos estudios muestran una gran variedad presente en toda Europa que permite concluir que la armonización de diferentes enfoques y formas sobre el doctorado pasan por la autonomía universitaria con lo cual, la contextualización del doctorado no ha de observarse desde un patrón único, aunque sí se reconozcan procedimientos comunes. Cada Estado miembro organiza el doctorado y las universidades construyen propuestas formativas a partir de sus principios pedagógicos.

Palabras clave: grado de Doctor, enseñanza superior, universidad, política educacional, reforma de la educación.

\begin{abstract}
This article presents a research on Doctorate in Europe with the aim of analyzing its harmonization according to the guiding criteria promoted by the European Higher Education Area (EHEA). Harmonization refers the recognition of university studies by all the signatory states based on procedural guidelines for curriculum administration. However, some differences appear: how the students are considered, length of study, and evaluation. This research was carried out according to a qualitative perspective to infer, analyze and interpret the meaning of the documents. Status of students registered in a doctoral degree and evaluation of these studies evidence a great variety in all Europe. It is concluded that the harmonization of different approaches and ways of developing the doctorate depend on the university autonomy. Therefore, the contextualization of the doctorate should not be observed from a unique pattern, although common procedures are recognized. Every state plans the doctorate and the universities construct training proposals based on their pedagogical principles.
\end{abstract}

Keywords: doctoral degree, higher education, universities, educational policy, educational reform. 


\section{INTRODUCCIÓN}

En términos curriculares, el doctorado es el último ciclo en los estudios universitarios. De acuerdo con lo que se ha establecido en el Espacio Europeo de Educación Superior (en adelante, EEES), son tres los ciclos: el primero es el grado, el segundo es el máster y el tercero es el doctorado, con lo cual, al finalizar los estudios de tercer ciclo se obtiene el título de doctor (Gijón y Crisol, 2012).

La organización de las enseñanzas cuenta con adaptaciones y particularidades según los sistemas universitarios de cada país y la autonomía universitaria presente en cada casa de estudios. No obstante, la forma en la que se organizan los estudios de doctorado, el sistema de evaluación, las etapas o compromisos formativos, tanto anuales como globales, se constituyen en el currículum de los estudios doctorales. Por lo tanto, al ser una estructura formal, organizada didácticamente, se habla lógicamente de su currículum.

La naturaleza curricular propia de cada universidad y contexto es una oportunidad para asumir la responsabilidad del sector universitario respecto al desarrollo social de cada país. Ahora bien, el concepto de armonización tiene ciertas imprecisiones, ya que no ha quedado claro si consiste en compartir orientaciones relativamente flexibles que han de aplicarse en todas las universidades de Europa o si se trata de unificar procedimientos administrativos de corte curricular.

Así, resulta útil identificar hasta qué punto la idea de la armonización ha supues- to una configuración relativamente uniforme en los estudios de tercer ciclo, ¿en todos los países europeos se considera de la misma manera a los estudiantes de doctorado?, ¿existe una duración más o menos uniforme respecto a los estudios doctorales?, ¿cómo se evalúan estos estudios? ¿hay exámenes generales a nivel de doctorado o solo es la defensa de la Tesis Doctoral la única actividad evaluativa?

En el EEES se ha asumido que el estudiante es el centro de atención. Esto significa que todo cuanto se haga debe ir enfocado hacia la mejora de la formación desde la organización de las enseñanzas doctorales para que estas sean diseñadas conforme a ciertos principios orientadores. Esto debe ayudar a responder a la hipótesis de que en los países pertenecientes al EEES, la administración de los estudios de doctorado recibe uniformemente el impacto de la armonización de las enseñanzas. Para intentar responder a esta hipótesis se observa el estatus de los matriculados en los estudios de tercer ciclo y los criterios generales para la evaluación en los estudios de doctorado en Europa como algunos de los principales indicadores que permiten estudiar, por ejemplo, similitudes entre los sistemas universitarios o las tendencias más notables respecto a la evaluación en el doctorado. A lo largo de este artículo se emplearán de manera indistinta los siguientes términos haciendo referencia al mismo sentido: estudios doctorales, doctorado y estudios de tercer ciclo. 


\section{Los estudios de doctorado. Algunas constataciones}

Los estudios de doctorado cuentan con un currículum propio de esta etapa de estudios (Vila et al., 2016) y en el EEES, según la Guía de Uso del Sistema Europeo de Transferencia y Acumulación de Créditos (en adelante, ECTS, por sus siglas en inglés), se señala que en Europa existen algunos países o instituciones en los que "el sistema ECTS se utiliza también en el tercer ciclo" (Comisión Europea, 2015, p. 27).

El ECTS se asigna "a todo el programa de doctorado o a algunos o a todos los componentes educativos (por ejemplo, unidades del curso impartidas)" (Comisión Europea, 2015 , p. 27). Se trata entonces de la organización curricular de los estudios de tercer ciclo. De acuerdo con Fernández y Wainerman (2015), se puede identificar dos momentos dentro de los estudios de doctorado. El primero tiene que ver con una aproximación inicial a toda una gama de posibilidades metodológicas y teóricas que permitan al doctorando familiarizarse con la profundidad del campo de estudio. El segundo momento está vinculado a la generación de la Tesis Doctoral (en adelante TD) y a todos los elementos que entran en juego para tal fin.

Se asume que una persona con el grado de doctor está cualificada para llevar a cabo una investigación de forma independiente, aplicando una serie de técnicas e instrumentos (Giudice, 2015). Además, el doctorado es "la titulación más emblemática de la universidad europea" (Haug, 2105, p.31). Así, tanto la tradición de este nivel académico como la visión que se tiene de este, merecen un tratamiento singular por cuanto el doctorado "se reconoce en la Declaración de Bo- lonia como un vehículo fundamental entre la docencia y la investigación y una herramienta básica para la relación con la sociedad" (Bermúdez et al., 2009, p. 194).

Lo anterior no impide reconocer que los países miembros han impulsado iniciativas de cambio en sus sistemas universitarios a nivel doctoral, por ejemplo, la creación de las escuelas de doctorado o el fomento de los equipos de investigación (Haug, 2015). Sin embargo, como se expondrá más adelante, aún hay notables diferencias entre un sistema universitario y otro. Es más, "en la mayoría de los casos encontramos una diferenciación cada vez mayor entre un doctorado de investigación y un doctorado profesional" (Bao et al., 2018, p. 19). En este sentido, el doctorado de investigación responde al interés de dedicarse a la carrera académica, mientras que el doctorado profesional se enfoca en la mejora de la práctica por medio de soluciones a problemas específicos.

La afirmación anterior responde al hecho de que en la sociedad del conocimiento se hace fundamental entender un diseño de estudios doctorales desde una visión transdisciplinaria como oportunidad privilegiada para el encuentro entre las diferentes áreas de estudio (Vila et al., 2016). El efecto contrario de esto es criticado por Mires (2014) al afirmar que "hay universidades en las que suelen formarse grupos cerrados que rinden culto a una determinada línea, excluyendo cualquiera posibilidad de interrelación con otras disciplinas del pensamiento" (p. 10).

En algunas investigaciones se observan coincidencias en cuanto a reconocer la rele- 
vancia del doctorado para el desarrollo del sistema educativo, la productividad científica, el compromiso social y la transformación del conocimiento en valor económico (Baeza, 2017; Buela-Casal et al., 2011; Castro y Sánchez, 2016; García-Peñalvo, 2016; López et al., 2018; Pacheco, 2015; Rolón et al., 2016; Silva-Cañaveral, 2016). Así, se acepta que el doctorado debe contar con una atención privilegiada que pueda garantizar la vinculación entre estos estudios y el desarrollo científico de los países.

A propósito de la idea de Giudice (2015), desde una perspectiva más enfocada hacia la sociedad del conocimiento, aquella persona que cuente con un doctorado debe tener competencias sobre la esfera investigativa, pero también debe contar con capacidad para relacionarse y trabajar, por ejemplo, con empresarios, investigadores de otras profesiones, representantes públicos o agentes políticos. Visto así, estamos ante una concepción de los estudios doctorales que va más allá de los linderos eminentemente investigativos (Haug, 2015; Patiño, 2019).
En este sentido, sí que existen ciertas diferencias de perspectiva en cuanto a la visión que se tiene sobre el doctorado. Para García-Peñalvo (2016) y Giudice Baca (2015), el doctorado no solo faculta para la investigación, sino que se ha de hacer frente a una serie de retos complejos que impone la sociedad del conocimiento y como ese conocimiento ha de revertirse en beneficio de la sociedad. Gijón y Crisol (2012) y Pacheco (2015) exponen que los estudios de doctorado constituyen una experiencia formativa de alto nivel que gira en torno a la investigación y el acercamiento a competencias investigativas y a la densidad teórica de un campo de estudio determinado. Aunque se asocie el grado de Doctor con una posible carrera académica, no siempre los doctorandos tienen este interés, por lo tanto, la trayectoria formativa de estos estudios no debería obviar las necesidades competenciales requeridas en el contexto laboral (Haug, 2015, p. 32). Aunque existan diferencias sobre el doctorado, a nivel general se proyecta tanto hacia la formación de investigadores como a la generación de conocimiento al más alto nivel (Fernández y Wainerman, 2015; Pacheco, 2015).

\section{LA IDEA DE LA ARMONIZACIÓN}

口-

El EEES unifica criterios que han de ser adoptados por los países signatarios del mismo. Esta armonización tiene como raíz la Declaración de la Sorbona (1998) en la que se expone la necesidad de unir esfuerzos para establecer líneas comunes que permitan una dinámica inclusiva dentro del contexto universitario europeo. Esta idea se refuerza con lo que expuso el Convenio de Lisboa (1997) respecto a reconocer los estudios en Europa para facilitar la movilidad académi- ca, por lo cual se establece que cada parte "adoptará las medidas necesarias para la aplicación de sus disposiciones en su territorio" (Convenio de Lisboa, 1997, art. II.1).

Ahora bien, ¿es obligatoria la armonización en el contexto universitario europeo? Esta interrogante puede responderse a partir de varias perspectivas. Primeramente, los países suscriben un acuerdo común y se comprometen a asumir los principios 
que allí se recogen (Alonso-Sáez y Arandia-Loroño, 2017; Álvarez et al., 2014), con lo cual se parte del punto de que, en efecto, la armonización forma parte indivisible del acuerdo del EEES. Desde otro punto de vista, se puede entender desde el interés por establecer una misma forma de funcionar, lo cual estaría opuesto al principio de autonomía universitaria ya que cada casa de estudios goza de libertad académica bajo ciertos estándares.

Estas connotaciones permiten inferir que la idea de la armonización es un tanto ambigua ya que, en la práctica, la frontera entre los acuerdos a nivel continental y la naturaleza autonómica de la institución universitaria puede verse comprometida ya que al ser una política pública, se ha expresado posiblemente de una forma que permite múltiples interpretaciones. Ahora bien, al estar en sintonía con los criterios orientadores del EEES, esto implica que desde la administración curricular se asumen ciertos elementos que se espera posibiliten el reconocimiento de los estudios en todo el EEES como, por ejemplo, el establecimiento del ECTS (Naval, et al., 2006) como unidad de medida de los créditos para calcular los tiempos de dedicación por parte del estudiante, solo que un crédito ECTS no supone la misma cantidad de horas en todos los países. Igualmente ocurre con la TD, siendo un requisito común, tiene formas diferentes a partir de los diseños curriculares de las universidades.

Otro de los aspectos vinculados a la idea de la armonización es que el estudiante pasa a ser el centro de atención de la universidad, pero al igual que con los demás elementos vinculados a la administración y diseño del currículum del tercer ciclo, no todos los países consideran de igual forma al estudiante de doctorado. Este hecho no deja de ser determinante por cuanto Europa fomenta que el estudiante es sujeto y no objeto de la educación, por lo tanto, es un actor activo y desde el momento en que se le considere empleado o usuario, se limita la participación determinante del estudiante con voz activa respecto a su propia formación, sin dejar de reconocer la relevancia que tiene este nivel de estudios para el desarrollo social y económico.

Además, la armonización pasa por el diseño de los programas doctorales y la evaluación del progreso de los doctorandos (Escudero y Trillo, 2015). Efectivamente, desde la autonomía universitaria se establecen los criterios bajo los cuales se ha de realizar la evaluación de los estudios de tercer ciclo, pero desde el EEES se persigue la idea del reconocimiento de los estudios y para que ese reconocimiento se logre, deben acordarse ciertos principios curriculares comunes para alcanzar la movilidad académica. En la realidad, la TD es el único requisito en común y a partir de ella existen diferentes organizaciones en cuanto a evaluación y estudios doctorales en todo el EEES. Si bien es cierto que esta variedad es el resultado de la diversidad institucional, social, histórica y contextual, también es cierto que dificulta la movilidad académica y el reconocimiento de los estudios dentro del EEES. Por lo tanto, la idea de la armonización pareciera más bien que es una invitación a un diálogo colectivo en la universidad europea, que se organiza bajo ciertos principios orientadores, pero que no supone una réplica exacta de maneras de funcionar (Naval et al., 2006).

¿A quién beneficia la armonización de los estudios doctorales en el EEES? Esta interrogante puede imprimir más complejidad a la comprensión de la idea de 
la armonización. Al parecer, y como resultado de la revisión de las declaraciones oficiales sobre el EEES, la armonización se maneja en términos poco precisos que dan pie a varias interpretaciones y formas de abordarla en la práctica. Además, se encuentra el desarrollo desigual de estudios doctorales, es decir, el área de las ciencias experimentales y la tecnología ha sido preferentemente privilegiada ya que, al contar y necesitar en muchos casos con inversión privada, se ha destinado expresamente al fomento de la investigación que los financiadores han necesitado (Buela-Casal et al., 2011, Jiménez, 2017; Rodríguez, 2018); mientras tanto, las ciencias sociales y las humanidades han tenido proporcionalmente una financiación a menor escala. El interés por armonizar todo un contexto universitario de alcance continental difícilmente puede lograrse en su totalidad (Cárdenas, 2015) porque cada Estado tiene su visión, intereses, necesidades y hasta limitaciones en cuanto al fomento de los estudios en este caso doctorales.

Se debe tener en cuenta si el camino andado hasta ahora ha favorecido la movilidad académica, el intercambio interinstitucional o el reconocimiento de los logros en términos curriculares de los estudiantes toda vez que, en un contexto en permanente cambio, un currículo estático es incompatible sin dejar de lado el hecho de que se trata de avanzar en una propuesta de cambio, tanto conceptual como metodológico, respecto a la forma de aprender (Olmedo, 2013). Tal y como exponen De Pablos Pons et al., (2007), en el EEES se "pretende aumentar la compatibilidad y la comparabilidad de los sistemas de educación superior europeos de manera coordinada sin menoscabo de la diversidad y especificidad de la oferta formativa universitaria, mediante la mejora de la calidad y la competitividad" (p. 534). La armonización debe contar con un conjunto de indicadores que puedan evidenciar hasta qué punto y en qué medida este acuerdo ha sido una realidad en función de la europeización de los estudios de tercer ciclo, tomando en consideración que la instauración del EEES establece nuevas formas de relaciones entre universidades y entre los sistemas universitarios (Rodríguez, 2018). De allí la importancia de analizar la configuración de los estudios de doctorado en Europa, desde la revisión tanto del estatus de los estudiantes de doctorado, como la forma en la que se administra el currículum.

\section{MÉTODo}

Esta investigación es fundamentalmente cualitativa. Esto implica que el análisis de contenido expone el sentido que emerge de los textos para inferir su sentido sin dejar de reconocer el contexto en el que se generan. La unidad de muestreo estuvo en los estudios de doctorado en el EEES y la unidad de registro se identificó en: estudios $3^{\circ}$ ciclo, organización, desarrollo, tipos, país, duración, estatus de estudiantes, evaluación en el doctorado, definición, criterios orientadores. A partir de Díaz (2018), se distinguen las siguientes etapas que corresponden al análisis:

I) Preanálisis: organización de la información, una vez realizada la revisión preliminar de las fuentes primarias y secun- 
darias, con el fin de identificar posibles limitaciones o carencias en función del objeto de estudio. Esta fase incluyó la revisión de los documentos oficiales propios del EEES para identificar los criterios orientadores de los estudios de doctorado: Magna Charta Universitatum (1988), Convenio de Lisboa (1997), Declaración de La Sorbona (1998), Declaración de Bolonia (1999), Declaración de Praga (2001), Declaración de Berlín (2003), Comunicado de Bergen (2005), Comunicado de Londres (2007), Comunicado de Lovaina (2009), Declaración del Área Europea de Educación Superior Budapest-Vienna (2010), Comunicado de Bucarest (2012), Declaración del Cuarto Foro de Política de Bolonia, Ereván (2015) y el Comunicado de París (2018).

II) Análisis y descripción: revisión analítica de investigaciones científicas y base de datos de EURYDICE para identificar el estatus de los estudiantes de tercer ciclo y la forma de evaluar en el doctorado en los países del EEES: Albania, Austria, Bélgica (comunidades flamenca, germana y francesa), Bosnia y Herzegovina, Bulgaria, Croacia, Chipre, República Checa, Dinamarca, Estonia, Finlandia, Macedonia, Francia, Alemania, Grecia, Hungría, Islandia, Irlanda, Italia, Letonia, Liechtenstein, Luxemburgo, Malta, Montenegro, Países Bajos, Noruega, Polonia, Portugal, Rumanía, Serbia, Eslovaquia, Eslovenia, España, Suecia, Turquía y Reino Unido.

III) Interpretación de los hallazgos de la investigación y elaboración de la conclusión. Los resultados se compararon a partir de las fuentes obtenidas para verificar su validez. De allí la importancia de identificar las fases de la investigación (tabla 1) para que los datos cualitativos puedan responder a los dos elementos de especial observación en esta investigación.

\section{Tabla 1}

Fases de la investigación

\begin{tabular}{|c|c|c|c|c|}
\hline FASES & $\begin{array}{l}\text { Estrategia de } \\
\text { recolección de datos }\end{array}$ & $\begin{array}{l}\text { Estrategia } \\
\text { sanalítica }\end{array}$ & Unidad de registro & Resultados \\
\hline I & $\begin{array}{l}\text { Revisión bibliográfica } \\
\text { Muestreo teórico } \\
\text { Revisión de documen- } \\
\text { tos oficiales del EEES }\end{array}$ & $\begin{array}{l}\text { Análisis inter- } \\
\text { pretativo y de } \\
\text { contenido } \\
\text { Análisis de con- } \\
\text { tenido }\end{array}$ & $\begin{array}{l}\text { Estudios } 3^{\circ} \text { ciclo } \\
\text { Organización } \\
\text { Desarrollo } \\
\text { Tipos } \\
\text { Definición } \\
\text { Criterios orientadores }\end{array}$ & $\begin{array}{l}\text { Fundamentación } \\
\text { teórica } \\
\text { Descubrir elemen- } \\
\text { tos de estudios de } \\
3^{\circ} \text { ciclo en docu- } \\
\text { mentos oficiales } \\
\end{array}$ \\
\hline II & $\begin{array}{l}\text { Análisis de bases de } \\
\text { datos de EURYDICE }\end{array}$ & $\begin{array}{l}\text { Revisión de } 36 \\
\text { países signata- } \\
\text { rios del EEES } \\
\text { Comparación de } \\
\text { los datos }\end{array}$ & $\begin{array}{l}\text { País } \\
\text { Duración } \\
\text { Estatus de estudiantes } \\
\text { Evaluación en el doc- } \\
\text { torado }\end{array}$ & $\begin{array}{l}\text { Configuración del } \\
3^{\circ} \text { ciclo en el EEES }\end{array}$ \\
\hline III & $\begin{array}{l}\text { Análisis e interpreta- } \\
\text { ción de los hallazgos } \\
\text { de la investigación, } \\
\text { opinión de expertos }\end{array}$ & & & $\begin{array}{l}\text { Informe de investi- } \\
\text { gación } \\
\text { Conclusiones }\end{array}$ \\
\hline
\end{tabular}

Nota: tabla de elaboración propia (2021). 


\section{RESUlTADOS}

Los resultados permiten inferir que armonización no es sinónimo de igualdad inequívoca respecto a los estudios de tercer ciclo (Naval et al., 2006; De Pablos Pons et al., 2007). Reconocer la diversidad de los sistemas universitarios europeos (Álvarez et al., 2014) debería ser una oportunidad para un mayor impacto en cuanto a la movilidad de estudiantes y de profesores. Sin embargo, no existe un único criterio sobre la organización de los estudios doctorales y las líneas de acción; no en todos los casos parecen ser las más favorecedoras.

Al explorar el EEES, se evidencia que los matriculados no tienen el mismo estatus en todos los países. En algunos casos, se les considera estudiantes y en otros se les considera empleados una vez que, siendo estudiantes, se les contrata para desarrollar actividades investigativas. En el caso de los estudiantes becados, ya sea de Europa o de fuera de ella, tienen el mismo tratamiento que los estudiantes originarios del EEES, según la política de cada país, solo que algunas cláusulas contractuales implican algún tipo de actividad determinada como publicación de artículos científicos, horas de docencia, entre otros. En todo caso, desde un punto de vista administrativo se les considera igual que al resto de matriculados.

\section{Tabla 2}

Países y grupo según estatus de los matriculados en estudios de $3^{\circ}$ ciclo - Europa

\section{Países}

Grupo

Austria, Bélgica (comunidad flamenca, comunidad alemana), Bosnia y Herzegovina, Bulgaria, Croacia, Chipre, República Checa, Estonia, Macedonia, Francia, Alemania, Grecia, Hungría, Islandia, Irlanda, Italia, Letonia, Liechtenstein, Lituania, Luxemburgo, Malta, Montenegro, Rumanía, Serbia, Eslovaquia, España, Suecia, Suiza, Turquía, Reino Unido.

Austria, Bélgica (comunidad flamenca), Dinamarca, Finlandia, Alemania, Países Bajos, Noruega, Serbia, Reino Unido.

Albania, Polonia, Portugal, Eslovenia, Suiza.

Nota: Elaboración propia (2020) con datos revisados y tomados de European Commision/EACEA/Eurydice (2018).

Para comprender estas proporciones, se organizaron los datos en tres grupos: el grupo A, países que consideran estudiantes a los matriculados en estudios de tercer ciclo; el grupo B, países que consideran empleados a los matriculados en estudios de tercer ciclo; y el grupo $\mathrm{C}$, países que no tienen un estatus específico. Se destaca que, en un mismo país, pueden coexistir varios estatus en los matriculados (Comisión Europea, 2018). Se asume que cuando un país se suscribe al EEES, se compromete a hacer los cambios necesarios que supone el acuerdo, en este caso, se crean las llamadas "Escuelas de Doctorado" que funcionan en todas las universidades. Tal y como se señala en 
la guía ETCS, a la persona matriculada en la educación superior se le denomina estudiante (Comisión Europea, 2015), sin embargo, todavía coexisten programas de doctorado anteriores a la firma del acuerdo del EEES ya en proceso de extinción y los nuevos doctorados con las orientaciones europeas. Además, desde la identidad propia de cada universidad, se reconocen organizaciones de estudios de tercer ciclo que posiblemente no estén del todo adaptadas a las orientaciones europeas. Lo que sí es una instrucción uniforme en todo el EEES es que los estudios de tercer ciclo terminan con la elaboración y defensa de la TD. Esto no depende de las universidades, sino que los países signatarios han de asegurar que esto se realice a través de las agencias nacionales de aseguramiento de la calidad.

En el grupo A se observa que el $66 \%$ de los países miembros del EEES consideran como estudiantes a sus matriculados en estudios de tercer ciclo. Esto permite inferir que, al menos en teoría, la figura de "estudiante" conlleva dentro de sí cierto amparo de parte de la universidad para con sus doctorandos; visto así, no tendría mayor relevancia. No obstante, otra interpretación que puede acompañar este porcentaje es el hecho de que, al ser un estudiante, cuenta dentro del total de estudiantes de una universidad y, por lo tanto, al ser la universidad en Europa mayoritariamente de adscripción pública, se hace necesario que la asignación presupuestaria se corresponda con el número de sus estudiantes. Si se persigue que los programas de doctorado alcancen altos estándares de calidad, es más que imperativa la necesidad de asegurar tanto los recursos materiales como los académicos que formen parte de dichos programas.
El grupo B representa el menor porcentaje de los datos revisados (10\%). En efecto, en este grupo se incluyen aquellos países que consideran empleados a los matriculados en estudios de tercer ciclo. Esto tiene que ver con el reconocimiento del joven investigador como un trabajador al que se contrata. Según el análisis, lo más común es que las contrataciones tengan una duración determinada (Comisión Europea, 2018) que exige una serie de actividades de tipo administrativo y burocrático mientras se desarrolla la investigación. Curiosamente, mientras se puede llegar a considerar que la contratación laboral al estudiar el doctorado pueda convertirse en una oportunidad para ingresar a la carrera académica, llama la atención que tan solo el 10\% de los países consideran esta posibilidad $\mathrm{y}$, según las proporciones, pareciera que la tendencia es a la baja. Se destaca además que, en un mismo sistema universitario, pueden existir estudiantes de doctorado y empleados que siguen estudios de tercer ciclo, por ejemplo, en Austria, Bélgica (comunidad flamenca), Alemania, Luxemburgo, Serbia o Reino Unido.

El grupo C representa el $24 \%$ de los países que no tienen un estatus específico para los matriculados. En Albania se les denomina "candidato a doctor", Bélgica (solo comunidad francófona) no tiene estudios doctorales, Liechtenstein los llama "investigadores" o "estudiantes" de manera indistinta, Suiza y Turquía los llama “empleados" o "estudiantes", Portugal no tiene un estatus específico, y Eslovenia los llama "investigadores jóvenes" (Comisión Europea, 2017). A partir de la lectura de los documentos oficiales del EEES, no se instruye de forma taxativa la forma en la que se debe llamar a los estudiantes del tercer ciclo, de allí las diferencias observadas. Tal y como 
se ha mencionado, solo la guía ETCS indica que el estudiante es la persona matriculada en los estudios universitarios, pero no diferencia entre un estudiante de primer, segundo o tercer ciclo.

Según Cárdenas (2015), el progreso asincrónico en cuanto a la adecuación de los estudios doctorales por parte de los sistemas universitarios europeos impide integrar ciertas características procedimentales comunes. Si bien resulta necesario asumir líneas de acción para la organización de las enseñanzas universitarias (Meroño y Ruiz, 2006), esto no se visualiza del mismo modo en todos los sistemas universitarios. Al intentar organizar de forma curricular las horas que dedica un estudiante a sus estudios se identifica otro problema: ni en todos los estudios de doctorado se asumen los ECTS, ni en todos los casos se entiende la misma cantidad de horas.

Otro dato interesante es la existencia de varios tipos de doctorado en un mismo país. Dinamarca tiene dos doctorados: el de investigación y el industrial. Rumanía tiene doctorado científico y doctorado profesional en artes y deportes. Reino Unido tiene cuatro tipos: doctorado integrado, doctorado profesional, doctorado por publicación y doctorado por aprendizaje a distancia. Con respecto a la evaluación en los estudios de tercer ciclo también hay particularidades. Con excepción de la elaboración y defensa de la TD, son más las diferencias que las semejanzas con respecto a las pautas para la evaluación de los estudios de tercer ciclo en toda Europa. Estas particularidades se presentan en la tabla siguiente (Tabla 3).

\section{Tabla 3}

Criterios generales usados para la evaluación en los estudios doctorales en Europa.

\begin{tabular}{llc}
\hline País & Evaluación & Años \\
\hline Albania & Cada institución establece reglas generales para evaluar la TD. & $3-5$ \\
\hline Austria & $\begin{array}{l}\text { Exámenes de cada asignatura. TD (puede o no estar compuesta por cierto nú- } \\
\text { mero de artículos). }\end{array}$ & 3 \\
\hline Bélgica (Cfl) & Defensa pública de TD. & 4 \\
\hline Bélgica (Cgr) & Defensa pública de TD (ya sea en forma de Tesis personal o publicaciones). & $\begin{array}{c}\text { Según } \\
\text { contrato }\end{array}$ \\
\hline Bélgica (Cfr) & No tiene estudios de tercer ciclo. & s/i \\
\hline $\begin{array}{l}\text { Bosnia y } \\
\text { Herzegovina }\end{array}$ & Exámenes orales o escritos. Defensa pública de TD. & 3 \\
\hline Bulgaria & Superación de plan de estudios y defensa de una TD. & $3-4$ \\
\hline Croacia & Defensa pública de TD & 3 \\
\hline Chipre & $\begin{array}{l}\text { Mínimo 6 semestres de estudios. Aprobar examen de contenido sobre área del } \\
\text { dpto. Defensa de TD. }\end{array}$ & 3 \\
\hline República & $\begin{array}{l}\text { Horas de docencia obligatorias. Superar plan de estudios individual y evalua- } \\
\text { ción anual por comisión del doctorado. Defensa pública de TD. Superación de } \\
\text { examen estatal de doctorado. }\end{array}$ & $5-6$ \\
\hline Dinamarca & Evaluación anual del plan del doctorado. Defensa de la TD. & 3 \\
\hline Estonia & Evaluación anual del plan del doctorado. Defensa de la TD. & $3-4$ \\
\hline Finlandia & Evaluaciones continuas. Exámenes, informes, Defensa de TD. & 4 \\
\hline
\end{tabular}




\begin{tabular}{|c|c|c|}
\hline Macedonia & $\begin{array}{l}\text { Formación académica organizada /cursos profesionales y científicos. Proyecto } \\
\text { de investigación. Movilidad internacional (al menos } 7 \text { días). Publicaciones } \\
\text { científicas internacionales. Participación en conferencias internacionales. Crear/ } \\
\text { presentar públicamente TD. }\end{array}$ & 3 \\
\hline Francia & $\begin{array}{l}\text { Estudiantes de doctorado realizan su trabajo bajo el control del director de Te- } \\
\text { sis. Defensa pública de TD. }\end{array}$ & $3-4$ \\
\hline Alemania & Defensa pública de TD. Superar algunas asignaturas. & $\mathrm{n} / \mathrm{d}$ \\
\hline Grecia & $\begin{array}{l}\text { El comité evalúa la TD en términos de calidad, inclusión, originalidad y contri- } \\
\text { bución a la ciencia. }\end{array}$ & 3 \\
\hline Hungría & $\begin{array}{l}\text { Exámenes orales o escritos. Tareas, trabajos, entre otros. Defensa pública de } \\
\text { TD. }\end{array}$ & 5 \\
\hline Islandia & Seguir plan de investigación y defender públicamente TD. & $3-4$ \\
\hline Irlanda & Seguir plan de investigación y defender públicamente TD. & $3-4$ \\
\hline Italia & $\begin{array}{l}\text { Evaluación anual de cada año del doctorado. Presentación de autoinformes. } \\
\text { Defensa pública de TD. }\end{array}$ & 3 \\
\hline Letonia & $\begin{array}{l}\text { Defensa pública de TD. Puede consistir en disertación, grupo de trabajos temá- } \\
\text { ticos, o una monografía. }\end{array}$ & 3 \\
\hline Liechtenstein & Defensa pública de TD. & 3 \\
\hline Lituania & Seguir plan de investigación y defender públicamente TD. & $4-6$ \\
\hline Luxemburgo & Seguir plan de investigación y defender públicamente TD. & 3 \\
\hline Malta & $\begin{array}{l}100 \text { horas anuales de docencia. Defensa pública de TD previo seguimiento del } \\
\text { plan de investigación. }\end{array}$ & 3 \\
\hline Montenegro & Evaluación anual de actividades formativas. Defensa pública de TD. & 3 \\
\hline Países Bajos & Seguir plan de investigación y defensa pública de TD. & 3 \\
\hline Noruega & Impartición de una o más conferencias. Defensa pública de TD. & 3 \\
\hline Polonia & $\begin{array}{l}\text { Preparación y defensa de TD. Docencia en su propia universidad. Superar prue- } \\
\text { bas específicas de la temática del doctorado. }\end{array}$ & $3-4$ \\
\hline Portugal & $\begin{array}{l}\text { Normas relativas a la defensa pública de TD u otro trabajo y concesión de una } \\
\text { calificación final están reguladas por las universidades. }\end{array}$ & $3-4$ \\
\hline Rumanía & Evaluaciones continuas. Exámenes, informes, Defensa de TD. & $3-4$ \\
\hline Serbia & $\begin{array}{l}\text { Extensa investigación científica, publicación de artículos en revistas científicas. } \\
\text { TD parte final del doctorado, excepto en caso de programas de estudio de artes. }\end{array}$ & $3-4$ \\
\hline Eslovaquia & Evaluación anual. Defensa de TD. & $3-4-5$ \\
\hline Eslovenia & Exámenes orales y escritos. Trabajos de investigación. Defensa pública de TD. & 3 \\
\hline España & $\begin{array}{l}\text { Evaluación del plan de investigación y portafolio de actividades personales del } \\
\text { doctorando. Defensa pública de TD. }\end{array}$ & $3-5$ \\
\hline Suecia & $\begin{array}{l}\text { Varias formas de evaluación continua. Necesario aprobar los cursos incluidos } \\
\text { en el programa y completar una TD de } 120 \text { créditos. }\end{array}$ & $3-4$ \\
\hline Turquía & $\begin{array}{l}\text { Examen de aptitud (oral y escrito), y de aprobar, se hace la defensa pública de } \\
\text { TD. }\end{array}$ & $2-3-4$ \\
\hline \multirow{2}{*}{ Reino Unido } & \multirow{2}{*}{ Defensa pública de TD. } & $3-4 y$ \\
\hline & & $5-7$ \\
\hline
\end{tabular}

Nota: tabla de elaboración propia (2020) con datos tomados de Comisión Europea /EACEA/Eurydice, 2017.

Con la idea de que la economía del conocimiento ha de fomentar una nueva relación entre las universidades y el sector pro- ductivo como oportunidad para fortalecer el desarrollo científico de los Estados (García-Peñalvo, 2016; López et al., 2018), el 
diseño de los estudios doctorales tiende más hacia lo organizativo-institucional (escuelas de doctorado) mientras que la sinergia entre investigación con pertinencia social y doctorado ha quedado bajo el amparo exclusivamente de cada sistema universitario, ya que la realidad política, económica, social y de acceso a los estudios es diversa. Por tanto, las necesidades que han de ser respondidas por la investigación doctoral no siempre tienen el mismo espectro de acción ni el mismo impacto, siendo el sector universitario un polo estratégico de desarrollo (García-Peñalvo, 2016; Giudice, 2015).

El requisito común es la elaboración y defensa de la TD. Tal y como se mencionó antes, la TD es el único aspecto obligatorio que se establece desde el EEES (Comisión Europea, 2017). Esto no depende de las universidades, más bien, son los países los que garantizan que esto se lleve a cabo a través de sus mecanismos de control. Sin embargo, existen particularidades en cuanto a la organización del contenido de la TD. Por ejemplo, en Dinamarca, Estonia y Finlandia se establece una evaluación anual del plan del Doctorado y su defensa; en Bélgica (Cfr) y Letonia, la TD puede hacerse con una disertación o una compilación de trabajos temáticos (generalmente artículos) relacionados con el tema de investigación.

Otros elementos de corte evaluativo se observan cuando se exige aprobar un examen general para superar los estudios de tercer ciclo, aparte de la elaboración de la correspondiente TD (Chipre, República Checa, Polonia, Eslovaquia y Turquía). También hay países en los que el período formativo corresponde con el estudio de asignaturas cuya aprobación dependerá del examen final de cada una de estas (Austria,
Bosnia y Herzegovina, Finlandia, Alemania, Hungría, Montenegro, Eslovenia). Al tratarse de estudios de tercer ciclo, la organización de esas asignaturas se hace desde "seminarios doctorales" que se evalúan con un examen. Con respecto a la docencia, no se ha observado exigencia expresa en cuanto a que los estudiantes de doctorado tengan la obligación de ejercerla, con excepción de República Checa, Malta y Polonia.

En la duración de los estudios también hay semejanzas y diferencias. La duración más común gira en torno a los 3-4 años. En Albania, República Checa, Hungría, Lituania, Eslovaquia y Reino Unido, la duración supera los 4 años, llegando a los 5, 6 y 7 años, en algunos casos. En el EEES, el principal requisito de acceso a los estudios de tercer ciclo es contar con un máster oficial (Comisión Europea, 2017); esto se cumple en todos los países miembros. Sin embargo, además de este requisito, cada país impone sus propios criterios.

En Albania y Serbia exigen el conocimiento de al menos un idioma de los 5 oficiales de la Unión Europea; en Austria, Bulgaria, Chipre, Estonia, Finlandia, Grecia, Liechtenstein, Lituania, Montenegro, Países Bajos, Noruega, Polonia, Portugal, Rumanía, Eslovaquia, Eslovenia y Suecia, los candidatos pasan por un proceso de admisión y selección competitiva diseñado por las propias universidades. En Macedonia, solo las cinco primeras instituciones de educación superior mejor calificadas del país pueden inscribir a nuevos candidatos de doctorado. Noruega exige además un contrato de trabajo como requisito. Respecto a las calificaciones de los estudios previos al doctorado, en Lituania, Serbia, Eslovenia, Suiza y Reino Unido se toma en consideración las 
calificaciones, especialmente, si se ha alcanzado al menos un título universitario con honores. En el caso español, al igual que en Bosnia y Herzegovina, como norma general se exige que los solicitantes hayan completado al menos 300 créditos del ECTS en estudios universitarios oficiales, de los cuales 60 deben pertenecer a un programa oficial de máster, además de los requisitos propios que cada universidad decida imponer.

La relación que tienen las universidades con los estudiantes es otro elemento para revisar. En efecto, aquellos que se han matriculado no reciben el mismo tratamiento y esto hace que la vinculación de estos con sus estudios doctorales no sea la misma.
Solo se ha alcanzado un acuerdo en cuanto a reconocer que se trata de estudios pertenecientes al último ciclo de las enseñanzas universitarias, pero no se ha logrado reconocer taxativamente que la necesidad de nuevas relaciones entre las universidades y la economía del conocimiento están dibujando nuevos estilos de doctorado (Bao et al., 2018). Las competencias doctorales deben ir más allá de lo exclusivamente investigativo (Giudice, 2015, Pacheco, 2015), ya que la interrelación de las disciplinas y el contacto laboral con otros actores de diferentes campos requieren habilidades que no se aportan solo desde la generación del conocimiento desvinculado de la realidad.

\section{DiscuSión Y CONCLUSIONES}

$\square$

Al menos en lo declarativo, la armonización persigue que todos los sistemas universitarios pertenecientes al EEES puedan contar con una estructura común que facilite la movilidad y el intercambio entre los miembros de la comunidad universitaria, por ejemplo, estudiantes y profesores (Bermúdez et al., 2009). Sin embargo, para que esto pueda llevarse a cabo se necesita unificar criterios de índole curricular y técnica que no siempre se adaptan a las realidades y particularidades de los países miembros, es más, puede darse el caso de que algunas orientaciones se acerquen a los linderos propios de la autonomía universitaria. En este caso, la ruta a seguir se hace aún complicada porque adoptar estándares de funcionamiento comunes para un continente tan diverso como el europeo es, cuanto menos, una labor compleja.
La armonización supone voluntad política e interés en discernir hasta qué punto es realmente necesaria. ¿Para qué sirve? ¿solo para garantizar la movilidad académica? Visto así, no tendría mucho sentido defenderla. Sería diferente si la armonización pretende la socialización del conocimiento no desde la competencia entre universidades sino desde la convicción de que el conocimiento es y debe ser para un mundo en constante cambio (Meroño y Ruiz, 2006). Como concepto, la armonización de los estudios de tercer ciclo tiende a ser flexible, de allí sus múltiples interpretaciones y la diversidad de formas de proceder.

Las diferencias observadas en los sistemas universitarios que se revisaron permiten afirmar que la armonización todavía es un proceso en construcción. En este sentido, desde el EEES se afianza la idea de que el es- 
tudiante se considera centro de atención de la dinámica universitaria, pero no todas las universidades europeas asumen este mismo principio, al menos en los estudios doctorales, lo cual implica que el modelo pedagógico de cada casa de estudio y de cada estado signatario, aunque válido, no se asocia completamente con este referente en cuanto al estudiante. Por otra parte, para lograr un reconocimiento de estudios a nivel continental, tanto el diseño como la administración del currículum y su gestión debe contar con elementos comunes y equiparables. Revisados los hallazgos de este estudio, se observan más diferencias que semejanzas en todo el EEES respecto a la configuración de los estudios de tercer ciclo, por lo tanto, no es posible un reconocimiento equiparable de tales estudios, al menos hasta el presente.

Desde una perspectiva comparativa, se observa que los matriculados en el tercer ciclo se consideran de forma diferente en todo el EEES ya que el nivel de impacto y la progresión en los estudios no se entienden de la misma manera, de allí que se observen dinámicas diferentes respecto a la forma de entender la evaluación en el doctorado. Solo existe un requisito común que es la TD.

Otro aspecto para resaltar es que todos los países del EEES tienen una agencia de calidad destinada a garantizar, entre otros elementos, el cumplimiento de los criterios que han de organizar las enseñanzas por parte de las universidades. Por ejemplo, la TD es un requisito uniforme en todo el EEES y esto es verificado por las agencias de calidad en cada universidad, pero las universidades, de acuerdo el principio de autonomía e identidad contextual, diseñan sus propuestas formativas y queda a criterio de las agencias la revisión de estas propuestas y su correspon- dencia con los principios del EEES. Por lo tanto, la autonomía universitaria se convierte efectivamente en un espacio para crear e impulsar modos de formación, pero siempre cuenta con la observación de las agencias de calidad que, a su vez, responden a la agencia europea de calidad para la educación superior.

El ejercicio investigativo realizado respecto a los autores permite confirmar, por una parte, la relevancia de los estudios doctorales para el desarrollo científico, económico y social precisamente en la actual sociedad del conocimiento (García-Peñalvo, 2016; López et al., 2018), pero, por otra parte, la armonización de los estudios doctorales tal y como se piensa que ha de realizarse, es muy difícil que se pueda conseguir (Cárdenas, 2015) debido a que cada Estado miembro tiene intereses, posibilidades y realidades socioeconómicas diferentes que pueden limitar la puesta en práctica de los cambios que se impulsan desde el EEES y que suponen una inversión importante para los Estados miembros (Bermúdez et al., 2009). Esto no es óbice para reconocer iniciativas de cooperación e intercambio entre universidades dentro del EEES.

Otra de las connotaciones de la armonización es que el progreso de los países respecto a los principios del EEES no ha ocurrido al mismo tiempo. Unos países llevan años de ventaja mientras que otros tienen un inicio cronológicamente posterior con lo cual, la equiparación de los estudios encuentra otro obstáculo. Hay países europeos que han dado un paso más en cuanto a la diversificación de los tipos de doctorado superando la visión monolítica de que el doctorado consiste solo en TD y artículos científicos (Haug, 2015; Patiño, 2019). 
Todo cambio en las enseñanzas universitarias debe pasar por reconocer el principio de adaptabilidad y flexibilidad curricular ya que la visión dogmática de las disciplinas no ayuda al acercamiento entre lo que se estudia y lo que el mundo espera (De Pablos Pons et al, 2007). Sería interesante continuar con investigaciones sobre la prospectiva de los estudios de tercer ciclo, sinergia entre el doctorado y la socialización del conocimiento, y el impacto de las investigaciones o las políticas para el fomento de los estudios doctorales. Se trata de innovar, crear y repensar nuevas lógicas en un mundo que atraviesa profundas y heterogéneas transformaciones.

\section{REFERENCIAS}

Alonso-Sáez, I., y Arandia-Loroño, M. (2017). 15 años desde la Declaración de Bolonia. Desarrollo, situación actual y retos del Espacio Europeo de Educación Superior. Revista iberoamericana de educación superior, 8(23), 199-213. https://bit.ly/33aqyor

Álvarez, A., González, J. A., Alonso, J., Arias, J. L., y Martís, J. R. (2014). Indicadores centinela para el plan de Bolonia. Revista De Investigación Educativa, 32(2), 327-338. https://cutt.ly/eyrXBvT

Baeza, P. (2017). Diversidad y diferenciación en la oferta de programas de doctorado en Chile. Calidad en la Educación, (47), 179-214. https://cutt.ly/wyrZ3x6

Bao, Y., Kehm, B., \& Ma, Y. (2018). From product to process. The reform of doctoral education in Europe and China. Studies in Higher Education, 43(3), 524-541. 10.1080/03075079.2016.1182 481

Bermúdez, M. P., Castro, Á., Sierra, J. C., y Buela-Casal, G. (2009). Análisis descriptivo transnacional de los estudios de doctorado en el EEES. Revista de Psicodidáctica, 14(2), 193-210. https:// bit.ly/2Q1L5pN

Buela-Casal, G., Guillén-Riquelme, A., Guglielmi, O., Quevedo-Blasco, R., y Ramiro, M. (2011). Rendimiento en el doctorado en función del área de conocimiento. Revista de Psicodidáctica, 16(1), 181-192. https://bit.ly/3cMLCpg

Cárdenas, J. (2015). La armonización en el Espacio Europeo de Educación Superior: El estado de la cuestión. Journal of Supranational Policies of Education, (1), 264-282. https://bit.ly/2vQg6pO

Castro, M. C., y Sánchez, M. (2016). La formación de investigadores en el área de humanidades: Los retos de la construcción de la voz autoral en la escritura de la tesis de doctorado. Revista signos, 49, 30-51. https://cutt.ly/CyrXe91

Comisión Europea. (2015). Guía de uso del ECTS. Luxemburgo: Oficina de Publicaciones de la Unión Europea. 10.2766/763090

Comisión Europea/EACEA/Eurydice. (2017). Modernización de la educación superior en Europa: personal académico - 2017. Informe de Eurydice. Luxemburgo: Oficina de Publicaciones de la Unión Europea. 10.2797/393811

Comisión Europea/EACEA/Eurydice. (2018). El Espacio Europeo de Educación Superior en 2018: Informe sobre la aplicación del Proceso de Bolonia. Luxemburgo: Oficina de Publicaciones de la Unión Europea. 10.2797/265898 
Comunicado de la Conferencia de Ministros Europeos responsables de Educación Superior. (2005). Comunicado de Bergen. https://cutt.ly/OtozjTb

Comunicado de la Conferencia de Ministros Europeos responsables de Educación Superior (2012). Comunicado de Bucarest: Aprovechando al máximo nuestro potencial: Consolidar el Espacio Europeo de Educación Superior. https://bit.ly/2VXcL35

Comunicado de la Conferencia de Ministros Europeos responsables de Educación Superior (2007). Comunicado de Londres: hacia el Espacio Europeo de Educación Superior: respondiendo a los desafios en un mundo globalizado https://bit.ly/2TAJgCm

Comunicado de la Conferencia de Ministros Europeos responsables de la Educación Superior (2009). Comunicado de Lovaina. https://bit.ly/2xrPbBr

Comunicado de la reunión de ministros europeos encargados de la educación superior (2001). Declaración de Praga: Hacia el Espacio Europeo de Educación Superior. https://bit.ly/2TFk2Df

Comunicado oficial de la Conferencia de Ministros responsables de Educación Superior (2003). Declaración de Berlín. https://cutt.ly/ztozlaG

Conferencia ministerial del Espacio Europeo de Educación Superior (2015). Declaración del Cuarto Foro de Politica de Bolonia Erevan https://bit.ly/2xqihB5

Conferencia Ministerial Europea de Educación Superior París (2018). Comunicado de París. https:// bit.ly/3cJ59Hb

Council of Europe. (1997). Convention on the recognition of qualifications concerning Higher Education in the European Region. https://cutt.ly/kyrXdnY

De Pablos Pons, J., Colás, P., González, T., y Jiménez, R. (2007). La adaptación de las universidades al Espacio Europeo de Educación Superior. Un proceso metodológico para el diseño de planes estratégicos. Revista De Investigación Educativa, 25(2), 533-554. https://bit.ly/2vQg8hq

Declaración conjunta de los ministros europeos de enseñanza (1999). Declaración de Bolonia. https://bit.ly/38E0eEl

Declaración Conjunta para la Armonización del Diseño del Sistema de Educación Superior Europeo a cargo de los Ministros Representantes de Francia, Alemania, Reino Unido e Italia (1998). Declaración de La Sorbona. https://bit.ly/2TBC215

Declaración de los rectores de las universidades europeas (1988). Magna Charta Universitatum. https://bit.ly/2Q0xsr0

Declaration on the European Higher Education Area (2010). Budapest-Viena, European Higher Education Area. https://bit.ly/2W0BymD

Díaz, C. (2018). Investigación cualitativa y análisis de contenido temático. Orientación intelectual de revista Universum. Revista general de información y documentación, 28(1), 119. https://cutt. ly/PjYuk9z

Escudero, J., y Trillo, F. (2015). Un análisis crítico del Espacio Europeo de Educación Superior como reforma de la enseñanza universitaria: los programas Verifica y Docentia en el contexto español. Educar em Revista, 31(57), 81-97. https://cutt.ly/myrXhpc

Fernández, L., y Wainerman, C. (2015). La dirección de tesis de doctorado: ¿una práctica pedagógi- 
ca? Perfiles educativos, 37(148), 156-171. https://cutt.ly/MyrXkfN

García-Peñalvo, F. (2016). La tercera misión. Education in the Knowledge Society, 17(1), 7-18. https://bit.ly/38Dt08b

Gijón, J., y Crisol, E. (2012). La Internacionalización de la Educación Superior. El caso del Espacio Europeo de Educación Superior. REDU. Revista de Docencia Universitaria, 10(1), 389-414. https://cutt.ly/5yrXK7D

Giudice, V. (2015). Competencias doctorales. Gestión En El Tercer Milenio, 18(35), 29-38. https:// bit.ly/332NN3T

Haug, E. (2015). Grados y másteres en España y el espacio europeo de educación superior (EEES). Conferencia de Rectores de las Universidades Españolas. https://bit.ly/2PZ108m

Jiménez, M. (2017). Convergencia europea e internacionalización del Doctorado: panorámica sobre las tendencias de cambio en España. Estudios pedagógicos (Valdivia), 43(1), 405-422. https:// cutt.ly/myrXGNv

López, D., Espinoza, O., y Sarzoza, S. (2018). Aplicación de políticas de aseguramiento de la calidad en programas doctorales. Opción, 34(86), 71-102. https://cutt.ly/Qta8cTr

Meroño, Á., y Ruiz, C. (2006). Estrategias de adaptación al Espacio Europeo de Educación Superior a partir del conocimiento y actitud del profesorado universitario. Revista De Investigación Educativa, 24(1), 281-298. https://bit.ly/2TzG1LF

Mires, F. (2014). La crisis de la Universidad. Paradigma. Revista de investigación educativa, 20(31), 9-18. https://cutt.ly/IyrXvdL

Naval, C., Sobrino, Á, y Pérez, C. (2006). La docencia universitaria ante el proceso de Bolonia. Revista panamericana de pedagogía, (8) 259-283. Recuperado de https://bit.ly/2TDjmxO

Olmedo, E. M. (2013). Enfoques de aprendizaje de los estudiantes y metodología docente: Evolución hacia el nuevo sistema de formación e interacción propuesta en el EEES. Revista De Investigación Educativa, 31(2), 429. https://cutt.ly/PyrXb9J

Pacheco, T. (2015). La tesis doctoral en ciencias sociales y su relación con el quehacer científico. Cinta de moebio, (52), 37-47. https://cutt.ly/MyrXmcB

Patiño, J. (2019). Análisis comparativo entre el doctorado profesional y de investigación en México. Revista iberoamericana de educación superior, 10(28), 25-41. https://cutt.ly/dyrXTau

Rodríguez, S. (2018). La Universidad: una visión desde "fuera" orientada al futuro. Revista De Investigación Educativa, 36(1), 15-38. https://cutt.ly/iyrXU1d

Rolón, V., Cabrera, C., y Ruffinelli, R. (2016). Gestión del conocimiento y la investigación científica en los cursos de Doctorado. Revista ScientiAmericana, 3(2), 1-16. Recuperado de https://cutt.ly/ Rta8v1h

Silva-Cañaveral, S. J. (2016). La investigación-creación en el contexto de la formación doctoral en diseño y creación en Colombia. Revista de investigación, desarrollo e innovación, 7(1), 49-61. 10.19053/20278306.v7.n1.2016.5601

Vila, D., Hernández, H., y Martínez, F. (2016). El diseño curricular doctoral desde la perspectiva transdisciplinaria. Revista Cubana de Educación Superior, 35(1), 114-129. https://bit.ly/2Q1atw9 